\title{
Neurovascular plexus theory for "escape pain phenomenon" in lower third molar surgery
}

\author{
Gururaj Arakeri', Mandeep Gill Sagoo' ${ }^{2}$, Peter A. Brennan ${ }^{3}$ \\ ${ }^{1}$ Department of Oral and Maxillofacial Surgery, Navodaya Dental College and Hospital, Raichur 584101, Karnataka, India. \\ ${ }^{2}$ Department of Anatomy and Human Sciences, King's College, London WC2R 2LS, UK. \\ ${ }^{3}$ Department of Oral and Maxillofacial Surgery, Queen Alexandra Hospital, Portsmouth PO6 3LY, UK.
}

Address for correspondence: Dr. Gururaj Arakeri, Department of Oral and Maxillofacial Surgery, Navodaya Dental College and Hospital, Raichur 584101, Karnataka, India. E-mail: gururaj.arakeri@gmail.com

\begin{abstract}
Pain during extraction of impacted mandibular third molars which can occur despite adequate local anesthesia is termed as "escape pain phenomenon". Recently, it was described during elevation of a mesioangular impacted mandibular third molar and also while curetting an extracted third molar socket. This phenomenon has been overlooked, as it was previously considered secondary to pressure effect on the inferior alveolar neurovascular bundle (IANB). However, it is unlikely that the pain impulses originate from direct pressure on the IANB, as the nerve is blocked more proximally at its entry into the mandible. The authors speculated that the occasional presence of a neurovascular plexus (NVP) independent of the IANB causes the escape of a pain impulse upon stimulation by root pressure or instrumentation. To validate the presence of such a plexus, a meticulous literature search and review were performed. The search revealed evidence of the occasional presence of a NVP consisting of auriculotemporal and/or retromolar neural filaments. The plexus may be present around the inferior alveolar artery or embedded within the IANB, and does not innervate the tooth. This plexus likely propagates pain impulses only upon stimulation by compression or instrumentation in the apical area of the tooth socket. This theory explains the absence of pain during tooth sectioning and bone guttering in the presence of a complete inferior alveolar nerve block.
\end{abstract}

Key words:

Inferior alveolar nerve, inferior dental plexus, escape pain phenomenon, third molar surgery

\section{INTRODUCTION}

The concept of the "escape pain phenomenon" (EPP) was described first by Carter and Keen ${ }^{[1]}$ in $4-5 \%$ of patients following an inferior alveolar nerve block. This phenomenon was observed during the entire course of the extraction procedure..$^{[1]}$ Recently, a concern was raised regarding the incidence of pain upon the elevation of an impacted third molar. ${ }^{[2,3]}$ The pain typically manifested

\begin{tabular}{|l|l|}
\hline \multicolumn{2}{|c|}{ Access this article online } \\
\hline Quick Response Code: & Website: \\
\hline & www.parjournal.net \\
\cline { 1 - 2 } & \\
\hline & Dol: \\
\hline
\end{tabular}

during elevation of the tooth and even during curettage of the extraction socket at the apical region. This pain was thought to be an alert for the proximity of root apices to the inferior alveolar canal. ${ }^{[2]}$ However, the pain was absent during soft tissue retraction, bone guttering, and tooth section procedures. ${ }^{[3]}$

The purpose of the present article was to postulate a theory which explains the EPP while elevation or curettage steps of third molar surgery based on a systematic literature search.

A literature search was conducted through the MEDLINE database using PubMed Central, Science Direct Search, Scopus, and Google. The keywords "neurovascular plexus" (NVP), "lower third molar", "inferior dental artery", "inferior alveolar nerve", "variation", "impaction and neurovascular complications" were used in all combinations. All papers are scrutinized for relevancy by a 
panel involving three staffs from the Anatomy, Physiology and Oral and Maxillofacial Departments. Content from the relevant papers was tabulated for analysis.

A total of six relevant papers (cadaveric studies on third molar innervations) were selected, and findings from the papers were recorded. The relevant information from each of these cadaveric studies is summarized below.

Carter and Keen ${ }^{[1]}$ noted a fine network of neurovascular bundles in the area lateral to the roots of the mandibular molar teeth and extending up into the ramus. This network was traced backward to one or more foramina in the areas of insertion of the muscles of mastication. The most common connection occurred with bundles leaving the lateral pterygoid and temporal muscles. The neurovascular bundles leaving the temporalis muscle were traced to foramina in the retromolar fossa, where the lowest fibers of the temporalis gain their insertion. This part of the network ramified through the cancellous bone, and eventually established one or more obvious junctions with the main trunk of the inferior alveolar nerve, or with branches sent by the latter to the molar roots. Microscopically, several bundles of this posterior plexus and nerve fibers and blood vessels were consistently demonstrated. The largest of supplementary foramina (internal diameter $0-4 \mathrm{~mm}$ or greater) was most commonly seen in the retromolar fossa (one-third of the mandibles) and near the condyle (one-fifth of the mandibles). The foramina were commonly in or near the areas of insertion of the muscles of mastication, and probably transmitted the neurovascular bundles found on dissection. ${ }^{[1]}$ These "accessory" nerves formed a plexus in the cancellous bone of the ramus and the body of the mandible lateral to the molar roots and the inferior alveolar nerve. Branches of this plexus seemed to join either the inferior alveolar nerve or its molar branches. ${ }^{[1]}$ The links described are believed to offer an alternative escape route for pain impulses even after the effective blockade of inferior alveolar nerve at its entry into mandible.

Variations in the branching pattern or topographical relationships of the mandibular nerve often accounted for failure to obtain adequate local anesthesia for routine oral and dental procedures, and for unexpected injury to branches of the nerve during operation. ${ }^{[4]}$ In 2/20 dissections, Anil et al. ${ }^{[4]}$ noted the emergence of the auriculotemporal nerve from the posterior root of the mandibular nerve. They also observed a nerve originating from the auriculotemporal nerve and joining the inferior alveolar nerve on both sides just posterior to the maxillary artery. These two nerve branches and the mandibular nerve formed a loop reminiscent of the brachial plexus. ${ }^{[4]}$

In a study by Zoud and Doran, ${ }^{[5]}$ the main trunk of the inferior alveolar nerve exhibited a branching structure reminiscent of the brachial plexus of the upper limb. This plexus-like structure was compounded by delicate interweaving of the inferior alveolar artery. There were numerous communications between the individual components, including fine filaments to the auriculotemporal nerve both proximal and distal to its origin. In one of the specimens, a number of nerve fibers were observed entering the mandible via the retromolar fossa. A second "plexus" was located between the mandibular canal and the roots of the mandibular teeth, and was composed of small fine filaments which arose from the intramandibular plexus. These fine filaments appeared to enter the roots of the teeth on their lateral surfaces as well as at the apices. The relationship of the inferior alveolar artery to the nerve plexus was notable. Instead of the artery lying below the nerve in the main part of the bony canal, and then passing superior to the nerve in the distal part of the channel as is most often described, the nerve and artery formed an intertwined plexus throughout the canal. The NVP thus lay in a distinct bony canal which was observed as far as the mental foramen, but which disappeared distal to this point. ${ }^{[5]}$

Blanton and Jeske ${ }^{[6]}$ found branches of the mandibular division of the inferior alveolar nerve originating high in the infratemporal fossa and travelling to the base of the coronoid process (high and anterior to the mandibular foramen) to enter the mandible. These branches carried sensory innervations to the second and third molars. Branches of the mandibular division or of its inferior alveolar or buccal branches also noted to enter the mandible in the retromolar fossa area and to carry sensory fibers to the first and third molars. The better-documented of the accessory nerves includes the mylohyoid nerve, as well as branches of the mandibular division (V3) of the trigeminal nerve, all of which arise high in the cranium and enter the mandible each according to its own route. The incidence of mylohyoid innervation to the mandibular teeth is approximately $60 \%$. The mylohyoid nerve can arise from the inferior alveolar nerve anywhere from $5 \mathrm{~mm}$ to $23 \mathrm{~mm}$ proximal to the level of the mandibular foramen, and it enters the mandible at a point distal to the mandibular foramen. Therefore, deposition of local anesthetic in the vicinity of the mandibular foramen during the administration of an inferior nerve block often does not block the mylohyoid nerve. The authors ${ }^{[6]}$ recommended performing the mylohyoid nerve block in the vicinity of the retromental foramina.

Studies have reported the incidence of the retromolar foramen as $1.7 \%,{ }^{[7,8]} 7.7 \%,{ }^{[9]}$ and $19.5 \%$ in the general population, ${ }^{[10]} 23 \%$ in native populations of North America $^{[11]}$ and $21.9 \%$ in the Indian population. ${ }^{[12]}$ However, Bilecenoglu and Tuncer ${ }^{[12]}$ found an incidence of $25 \%$ which is the second highest rate in the literature after Schejtman et al. ${ }^{[13]}$ study (72\%). The histopathologic investigation found the contents of the neurovascular bundle to be striated muscle fibers, thin myelinated nerve fibers, numerous venules, and a muscular artery having a lumen of $120-130 \mu \mathrm{m}$. This is similar to the results found in Schejtman's studies. ${ }^{[13]}$ Compared to the nutrient foramina and canals, the retromolar foramen and canal were found to have vascular and neural contents. The presence of this type of canal may explain anesthetic insufficiency and/or bleeding at this location during routine surgery. ${ }^{[7]}$ The distal end of the retromolar canal advanced to the 
distal root of the third molar and retromolar area, and this distribution showed that the contents of this canal innervate and supplied the third molar and mucosa of the retromolar area.

Coleman and $\mathrm{Smith}^{[14]}$ speculated that aberrant nerve branches to the mandibular teeth and periodontium arising from major branches of the mandibular trunk high within the pterygomandibular space could also be bathed by anesthetic deposited at the mandibular neck. These branches would probably escape the drug when it is deposited at the mandibular foramen. The authors ${ }^{[14]}$ also cited Sutton's ${ }^{[15]}$ and Rood's ${ }^{[16]}$ papers which suggested that there may be accessory innervation of the mandibular teeth from branches of the lingual, buccal, facial, and upper cervical nerves from their clinical experience. With the exception of the buccal nerve, there is little anatomic evidence to support these opinions.

\section{Neurovascular plexus theory}

The above authors have demonstrated "accessory" nerves from the lateral pterygoid muscle, the temporal muscle, the auriculotemporal nerve, and the mylohyoid nerve. In most instances, these accessory nerves pass through foramina of the condylar neck, retromolar fossa, or within the infratemporal fossa to form a neural plexus which communicates with the inferior alveolar neurovascular bundle. However, all of the authors note that this accessory nerve or the plexus innervates the third molar. Conceptually, if this nerve plexus does, in fact, supply the third molar, then the pain would be expected from the commencement of tooth removal procedure, and not specifically during elevation of the tooth or socket curettage.

Based on the current literature search, the authors hypothesize that the EPP in lower third molar surgery can be attributed to the occasional presence of a NVP lying deep to the roots of the third molar which does not provide innervation. This plexus may be formed by various nerves including the auriculotemporal, mylohyoid, and retromolar plexus from the pterygoid and masseter

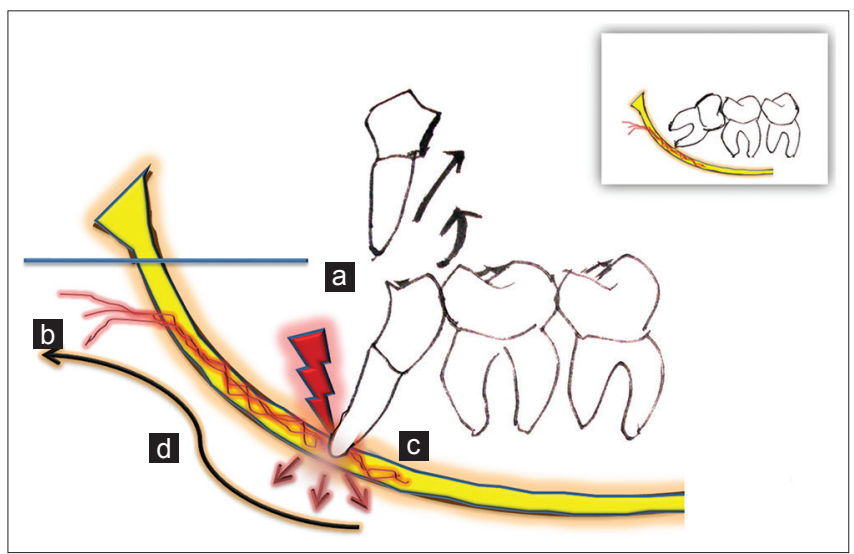

Figure 1: Diagrammatic illustration of escape pain phenomenon (EPP) and neurovascular plexus (NVP) theory during the two section technique of third molar removal (inner figure: mesioangular mandibular third molar impaction). (a) Conduction block; (b) neurovascular/nerve plexus entering into the bony canal; (c) compression of the neurovascular bundle; (d) escape of pain away from conduction blockade through neurovascular/nerve plexus muscles. The pain impulses may be generated upon compression or stimulation of the plexus and be carried away causing pain escape in the presence of a complete nerve block [Figure 1].

\section{DISCUSSION}

Since the early 1970 s, dentistry has experienced a resurgence of interest in the neuro-anatomical basis of local anesthesia, resulting in many scientific reports on the subject. ${ }^{[6,17]}$ Numerous studies have provided a detailed knowledge of the anatomy of the trigeminal nerve, which is important in obtaining profound local anesthesia. ${ }^{[18-21]}$ To explain the incidence of inadequate anesthesia in the mandibular region despite an efficient inferior alveolar nerve block, an EPP was first described by Carter and Keen. ${ }^{[1]}$ It was suggested to deposit local anesthetic solution in the vicinity of the retromandibular foramen to prevent the pain escape. ${ }^{[6]}$ However, the persistence of pain escape noted even after infiltrating the retromolar area with lidocaine solution in 5 of our cases. Recently, Ngeow $^{[2]}$ noted the incidence of EPP while the elevation of an impacted tooth and assumed it as a result of compression of inferior alveolar nerve. It was postulated that the release of sodium and potassium ions from the compressed nerve may be responsible for propagating the pain impulses. ${ }^{[2]}$ This hypothesis was criticized because the pressure would result in paresthesia which sustains long even after the procedure.

The theory based on present literature validated the presence of a plexus at the apical region of the tooth which may be stimulated by inadvertent tooth elevation or postextraction curettage.

The incidence of pain escape seems to occur only during third molar surgery because of the inclination impacted tooth, as well as the curvature of the angle of the mandible. This brings the neurovascular bundle in proximity to the tooth root. It is not seen in the first and second molar regions as there is no possibility of compression of the neurovascular bundle.

There was also a significant incidence of bleeding following the EPP, which usually noted immediately following the elevation of the tooth fragment. This may be secondary to damage inferior alveolar vessels or vessels from the NVP.

A large-scale cadaveric study would confirm the presence of these independent NVP and their third molar innervations.

\section{REFERENCES}

I. Carter RB, Keen EN. The intramandibular course of the inferior alveolar nerve. J Anat 197I; 108:433-40.

2. Ngeow WC. Tooth section technique for wisdom teeth. Int JOral Maxillofac Surg 2009;38:908.

3. Arakeri G, Arali V. Tooth section technique and pain upon elevation in third molar removal. Int J Oral Maxillofac Surg 2010;39:98-9.

4. Anil A, Peker T, Turgut HB, Gülekon IN, Liman F. Variations in the anatomy of the inferior alveolar nerve. Br J Oral Maxillofac Surg 2003;41:236-9. 
5. Zoud K, Doran GA. Microsurgical anatomy of the inferior alveolar neurovascular plexus. Surg Radiol Anat 1993;15:175-9.

6. Blanton PL, Jeske AH, ADA Council on Scientific Affairs, ADA Division of Science. The key to profound local anesthesia: neuroanatomy. J Am Dent Assoc 2003; 134:753-60.

7. Ossenberg NS. Temporal crest canal: case report and statistics on a rare mandibular variant. Oral Surg Oral Med Oral Pathol 1986;62:10-2.

8. Ossenberg NS. Retromolar foramen of the human mandible. Am J Phys Anthropol 1987;73:1 19-28.

9. Sawyer DR, Kiely ML. Retromolar foramen: a mandibular variant important to dentistry. Ann Dent 1991;50:16-8.

10. Kodera $\mathrm{H}$, Hashimoto I. A case of mandibular retromolar canal: elements of nerves and arteries in this canal. Kaibogaku Zasshi 1995;70:23-30.

II. Narayana K, Nayak UA, Ahmed WN, Bhat JG, Devaiah BA. The retromolar foramen and canal in South Indian dry mandibles. Eur J Anat 2002;6:141-6.

12. Bilecenoglu B, Tuncer N. Clinical and anatomical study of retromolar foramen and canal. J Oral Maxillofac Surg 2006;64: I493-7.

13. Schejtman R, Devoto FC, Arias NH. The origin and distribution of the elements of the human mandibular retromolar canal. Arch Oral Biol 1967; | 2:1261-8.

14. Coleman RD, Smith RA. The anatomy of mandibular anesthesia: review and analysis. Oral Surg Oral Med Oral Pathol 1982;54:148-53.

15. Sutton RN. The practical significance of mandibular accessory foramina. Aust Dent J 1974;19:167-73.
16. Rood JP. The analgesia and innervation of mandibular teeth. Br Dent J 1976; |40:237-9.

17. Zaytsev AY, Nazaryan DN, Kim SY, Dubrovin KV, Svetlov VA, Khovrin VV. Features of maxillary and mandibular nerves imaging during stem regional blockades. Anesteziol Reanimatol 2014;2:44-6.

18. Sinha P, Tamang BK, Sarda RK. Communication between Mylohyoid and Lingual Nerve: an anatomical variation. J Clin Diagn Res 2014;8:AD0 I-2.

19. Jing Q, Wan K, Wang XJ, Ma L. Effectiveness and safety of computer-controlled periodontal ligament injection system in endodontic access to the mandibular posterior teeth. Chin Med Sci J 20I4;29:23-7.

20. Tan VL, Andrawos A, Ghabriel MN, Townsend GC. Applied anatomy of the lingual nerve: relevance to dental anaesthesia. Arch Oral Biol 20I4;59:324-35.

21. Chiono J, Raux O, Bringuier S, Sola C, Bigorre M, Capdevila X, Dadure C. Bilateral suprazygomatic maxillary nerve block for cleft palate repair in children: a prospective, randomized, double-blind study versus placebo. Anesthesiology 2014;120:1362-9.

How to cite this article: Arakeri G, Sagoo MG, Brennan PA. Neurovascular plexus theory for "escape pain phenomenon" in lower third molar surgery. Plast Aesthet Res 2015;2:107-10.

Source of Support: Nil, Conflict of Interest: None declared.

Received: 10-11-2014; Accepted: 10-04-2015 